\title{
Correction: Differences in eye movement range based on age and gaze direction
}

\author{
Won June Lee ${ }^{1} \cdot$ Ji Hong Kim $\mathbb{D}^{1,2} \cdot$ Yong Un Shin ${ }^{1} \cdot$ Sunjin Hwang ${ }^{1} \cdot$ Han Woong Lim ${ }^{1}$
}

Published online: 28 March 2019

(c) The Royal College of Ophthalmologists 2019

\section{Correction to: Eye;}

https://doi.org/10.1038/s41433-019-0376-4; published online 5 March 2019.

Since the online publication of the above article, the authors have noted an error with the funding. The authors apologize for any convenience caused by this error.
The correct funding is:

This work was supported by the research fund of Hanyang University (HY-201700000000487) and the National Research Foundation of Korea (NRF) grant funded by the Korea government (MSIP) (No. 2016R1C1B2015164).

These authors contributed equally: Won June Lee, Ji Hong Kim

The original article can be found online at https://doi.org/10.1038/ s41433-019-0376-4.

Han Woong Lim

limhw@nate.com

1 Department of Ophthalmology, Hanyang University College of Medicine, Seoul, Korea

2 Department of Ophthalmology, Armed Forces Capital Hospital, Seongnam, Korea 